Reprod. Nutr. Dévelop., 1986, 26 (2 A), 417-427.

\title{
Micro-dosage automatisé de la céruloplasmine plasmatique par mesure de l'activité oxydasique chez les bovins et les ovins
}

\author{
J. P. CHACORNAC, J. BARNOUIN, T. RABOISSON
}

avec la collaboration technique de Marie-Françoise DEVINS (*)

Laboratoire d'Eco-Pathologie,

(*) Laboratoire des Maladies nutritionnelles, I.N.R.A., Theix 63122 Ceyrat, France.

Summary. An automated micro-method for the determination of ceruloplasmin in bovine and ovine plasma by measurement of oxidase activity.

An automated method for the determination of plasma ceruloplasmin in bovine and ovine species is described. The analytic principle of Holmberg and Laurell (1951), based on protein oxidase activity, has been applied to a discrete computorized analyzer (Isamat, Isa Biologie, 94230 Cachan, Francel.

Using this method a single operator can determine the oxidase activity of 500 plasma ceruloplasmin samples per day.

The method is accurate $(2.6 \%$ precision for mean values), linear over a range of $7-240 \mathrm{mcg} / 100 \mathrm{ml}$ in copper equivalents and low in cost because small volumes of reagents can be used; it also correlates well with results from standard atomic absorption spectrophotometry and single radial immunodiffusion methods (correlation coefficients : 0.944 to 0.968).

The conservation of oxidase activity is adequate and plasma ceruloplasmin oxidase activity determination can be used for assessing copper nutritional state and for screening inflammatory processes in bovine and ovine herds.

\section{Introduction.}

Le dosage de la céruloplasmine plasmatique chez les ruminants a un intérêt double : appréciation de l'état nutritionnel vis-à-vis du cuivre, $95 \%$ du cuivre alimentaire étant incorporé à la céruloplasmine (Mason et al., 1980 ; Auza, 1983) ; dépistage des animaux développant une réaction inflammatoire (Lamand et Levieux, 1981 ; Conforti et al., 1982).

Or, les risques d'états carentiels en cuivre ne sont pas à négliger en France (Lamand et al., 1973 ; Barnouin et al., 1985) et le dépistage précoce des états inflammatoires non spécifiques survenant dans les troupeaux est un critère utile 
d'appréciation de l'état de santé des animaux en vue d'une réponse thérapeutique rapide.

Mais le développement d'une technique diagnostique en pathologie animale est liée à sa facilité de mise en œuvre et à son faible coût : ces considérations nous ont donc amenés à mettre au point et à évaluer un micro-dosage automatisé de la céruloplasmine plasmatique, basé sur l'activité oxydasique de cette protéine, mise en évidence par Holmberg et Laurell (1951).

\section{Matériel et méthodes.}

\section{Animaux.}

1) Bovins. - 92 animaux sont utilisés (vaches, veaux, taurillons, taureaux) ; ils proviennent d'élevages de rente $(51$ sont cliniquement atteints et 41 indemnes d'états pathologiques inflammatoires) et sont prélevés dans le cadre d'une étude sur les paramètres de I'inflammation chez les bovins (Raboisson, 1985).

2) Ovins. - Dix animaux d'un an environ sont utilisés, soit 5 mâles et 5 femelles croisés Romanoff-Berrichon) ayant reçu une injection intradermique d'immunoglobulines de chèvre. Seize brebis provenant d'élevages de rente et cliniquement atteintes de diverses pathologies inflammatoires.

Pour les bovins et les ovins, le choix des animaux est effectué afin de faire des comparaisons de méthodes à partir d'échantillons ayant un grand domaine de variation.

\section{Prélèvements.}

1) Bovins. - Du sang est prélevé à la veine caudale dans un tube hépariné ; il est centrifugé immédiatement et les plasmas obtenus sont placés à $4^{\circ} \mathrm{C}$ puis congelés à $-20^{\circ} \mathrm{C}$ jusqu'à l'analyse, entre 2 et $8 \mathrm{~h}$ après le prélèvement.

2) Ovins. - Le sang est prélevé sur héparine à la veine jugulaire, le jour de l'injection d'immunoglobulines, ainsi que 5, 6, 7, 8 et 9 jours après l'injection pour le premier groupe d'animaux et le jour de l'observation de la pathologie, pour le second groupe. Les plasmas obtenus sont conservés à $-20^{\circ} \mathrm{C}$ jusqu'à l‘analyse.

\section{Analyses.}

1) Principes. - Pour la méthode dont la mise au point est l'objet de cette étude, on mesure l'activité oxydasique de la céruloplasmine à partir de l'oxydation de la para-phénylène diamine, les conditions optimales du développement de la réaction ayant été précisées chez l'homme par Jérôme et Girault (1969), chez I'homme et le rat par Sunderman et Nomoto (1970) et chez les ovins par Calabrese et al. (1983), notamment le $\mathrm{pH}$ de la réaction.

Pour évaluer le dosage de la céruloplasmine basé sur l'activité oxydasique $(\mathrm{OXY})$ on compare les résultats obtenus à ceux déterminés :

- par une méthode immunologique de dosage de la céruloplasmine utilisant l'immunodiffusion radiale (IDR), décrite par Mancini et al. (1965), à partir d'un antisérum préparé sur lapin (Levieux, non publié) ; 
- par dosage de la cuprémie plasmatique (CUP), décrit par Bellanger et Lamand (1975) et utilisant la spectrophotométrie d'absorption atomique.

2) Automate. - La détermination de l'activité oxydasique est réalisée sur un analyseur à transfert mono-canal informatisé Isamat (Isa-Biologie - 94230 Cachan, France) dont la description et l'évaluation ont été effectuées par Pressac et al. (1983).

\section{3) Réactifs.}

Réactif 1: $68 \mathrm{~g}$ d'acétate de sodium (Prolabo, Réf. : 27650.292) et $186 \mathrm{mg}$ d'éthylène diamine tétracétique (Prolabo, Réf. : 20302.293) sont dissouts dans 1 I d'eau bidistillée, la solution étant amenée à $\mathrm{pH} 8,9,10$ ou 11,5 suivant les essais avec $\mathrm{NaOH} 4 \%$; le pH 9 est choisi en définitive.

Réactif 2 : $250 \mathrm{mg}$ de para-phénylènediamine dichlorhydrate (Carlo Erba, Réf. : 45 0673) sont dissous dans $50 \mathrm{ml}$ d'eau bidistillée. A l'inverse de Jérôme et Girault (1969), nous n'avons pas choisi de tamponner le substrat ayant observé sa meilleure stabilité en solution non tamponnée (1 semaine) qu'en présence de tampon (1 jour).

Gamme étalon : la gamme est préparée à partir de plasmas ( 1 bovin, 1 ovin) à teneurs élevées en céruloplasmine, déterminées après étalonnage avec une solution de céruloplasmine bovine Sigma, Réf. C-2026. L'activité oxydasique de cette solution ( 3500 unités oxydasiques $/ \mathrm{ml}$ ou u.O) est définie à partir d'une unité arbitraire, soit la quantité " d'oxydase " entraînant une déviation optique de 0,01 par min à $550 \mathrm{mn}\left(\mathrm{pH} 6,4 ; 37^{\circ} \mathrm{C}\right)$ en présence d'un substrat le N,N-diméthyl-pphénylénédiamine. Cette solution de céruloplasmine titre par ailleurs $119 \mu \mathrm{g} / \mathrm{ml}$ de cuivre, déterminé par spectrophotométrie d'absorption atomique. Le mode d'étalonnage indirect est choisi au vu du coût très élevé de la solution de céruloplasmine de référence, qui ne permet pas son utilisation en routine dans la confection de la gamme d'étalonnage. Dans le cadre de la mise au point de la méthode, on a néanmoins utilisé cette solution pour évaluer la linéarité et la récupération. En fin de compte, les points de gamme qui sont utilisés titrent $O$ (blanc de gamme) ; 10,$7 ; 21,4$ et 42,8 u.O.

4) Mode opératoire. - Le dosage de la céruloplasmine est effectué à $37^{\circ} \mathrm{C}$. Le volume d'échantillon (plasma) utilisé par analyse est de $12 \mu \mathrm{l}$. L'échantillon est mélangé avec $200 \mu \mathrm{l}$ de réactif 1 , auxquels sont ajoutés, après 3 min d'incubation, $100 \mu \mathrm{l}$ de réactif 2 . Les paramètres du micro-ordinateur SIM/SILEX (IsaBiologie) sont fixés selon la figure 1 à partir d'une disquette 6 pouces « point terminal » (Isa Biologie, Réf. : 1.0.00.099). Le développement de la réaction est calculé à partir de deux lectures de densité optique (D.O) à $550 \mathrm{~nm}$, la première effectuée $289 \mathrm{~s}$, après adjonction du réactif 2 , la seconde $345 \mathrm{~s}$ après la première : cette variation de D.O est utilisée pour calculer l'activité oxydasique de la céruloplasmine.

La méthode permet la réalisation du dosage et l'affichage des résultats pour 26 échantillons en $15 \mathrm{~min}$, soit une cadence de 70 à 100 échantillons par heure pour un opérateur.

5) Mesures de la répétabilité et de la reproductibilité. - Elles sont réalisées à l'aide de plasmas de vache à activités oxydasiques dites " faibles ", " moyennes" 
et «fortes ", selon un protocole défini par la Société Française de Biologie Clinique (Grafmeyer, 1978, schéma 2).

FIG. 1. - Paramètres du micro-ordinateur SIM/SILEX pour la détermination de l'activité oxydasique de la céruloplasmine.

$\mathrm{Nb}$ de blancs

Le blanc est-il un étalon?

$\mathrm{Nb}$ d'étalons

Mode utilisé

$\mathrm{Nb}$ de tours de chauffage

$\mathrm{Nb}$ de tours d'attente

$\mathrm{Nb}$ de tours à ignorer

Préchauffage bras

Volume prélèvement \%

Volume diluant \%

Volume réactant Micro L

Vitesse seringue prélèvement

Vitesse seringue diluant

Limite inférieure blanc

Limite supérieure blanc

Coefficient conversion G-SI

$\mathrm{Nb}$ de décimales

Filtre

Température

Option $1: 0=$ NON, $1=$ OUI

\section{Résultats.}

1. Détermination du $\mathrm{pH}$ réactionnel optimal chez les bovins.

1.1. Linéarité à différents $p H$. - La linéarité de la méthode est evaluée à partir de solutions de céruloplasmine, à différents $\mathrm{pH}$ de tampon (réactif 1 ) soit 8,9 , 10 et 11,5. La méthode se révèle linéaire pour les $\mathrm{pH} 8,9$ et 10 (fig. 2) de 2,2 à $70 \mathrm{u} . \mathrm{O}$ (soit en correspondance cuivre de 7 à $240 \mu \mathrm{g} / 100 \mathrm{ml}$ ), les meilleurs coefficients de corrélation étant obtenus à $\mathrm{pH} 9$ et $10(0,9995$ et 0,9998$)$.

1.2. Répétabilité à différents $p H$. - La répétabilité est évaluée, pour les $\mathrm{pH}$ pour lesquels la méthode est linéaire. Le plus faible coefficient de variation moyen $(1,8 \%)$ est obtenu à $\mathrm{pH} 9$. A ce $\mathrm{pH}$, la valeur du plasma « faible » est de $17,3 \pm 0,8$ u.O ( $\mathrm{n}=6, \mathrm{C} . \mathrm{V}=4,6 \%$ ), celle du plasma " moyen " de $28,7 \pm 0$ u.O ( $n=3, \mathrm{C} . \mathrm{V}=0 \%$ ) et celle du « fort » de $55,7 \pm 0,5 \mathrm{u} . \mathrm{O}$ ( $\mathrm{n}=6$, $\mathrm{C} . \mathrm{V}=0,9 \%$ ). A pH 8 , le $\mathrm{C} . \mathrm{V}$ moyen obtenu est de $2,9 \%$, à $\mathrm{pH} 10$ il est de $2,2 \%$.

1.3. Pourcentage de récupération à différents $p H$. - Les tests de récupération (tabl. 1) sont effectués aux pH pour lesquels la méthode est linéaire à partir d'un plasma dosé 3 fois pour chaque $\mathrm{pH}$ dans la même série (valeurs: $36,2 \pm 0,6$ u. 0 à $\mathrm{pH} 8 ; 32,6 \pm 0,3$ à $\mathrm{pH} 9 ; 32,2 \pm 1,6$ à $\mathrm{pH} 10 ;$ ces valeurs sont considérées comme valeurs de référence).

Les plasmas sont ensuite dilués au 1/2 dans de l'eau bidistillée (modalité 1) puis on leur ajoute, à volume égal, des solutions de céruloplasmine à : 8,$7 ; 17,5$; $35 ; 53,8$ et 70 u.O (modalités 2 à 6 ). 


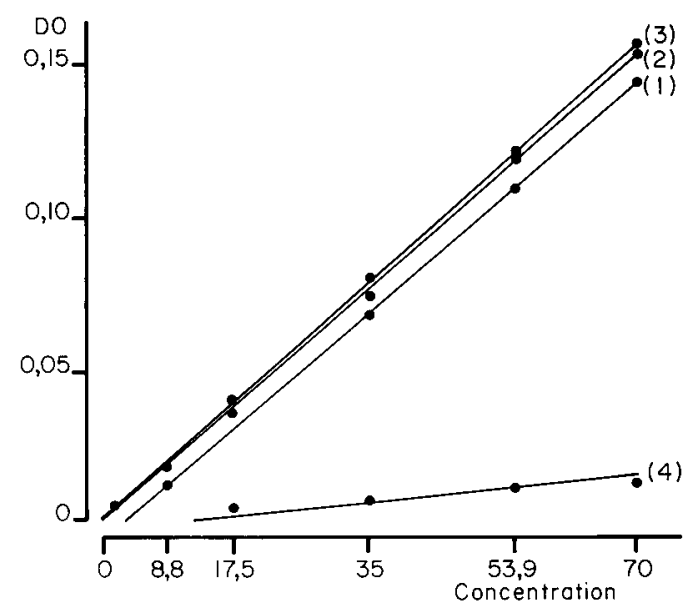

FIG. 2. - Linéarité de la méthode de détermination de l'activité oxydasique de la céruloplasmine en solution aqueuse à différents $\mathrm{pH}$ du tampon.

(1) $\mathrm{pH}$ du tampon: $8, r: 0,982, y=2,067 x+1,105\left(^{*}\right)$; (2) $\mathrm{pH}$ du tampon: $9, r: 1,000$, $Y=2,218 x-1,378$; (3) $p H$ du tampon : $10, r: 1,000, Y 22,239 x-0,134$; (4) pH du tampon : $11,5, r: 0,942, y=0,159 x-0,813$.

${ }^{*}{ }^{*}$ Coefficient de corrélation $(r)$ de la gamme et équation de la droite de régression.

\section{TABLEAU 1}

Résultats des tests de récupération.

\begin{tabular}{|c|c|c|c|c|c|}
\hline \multirow{2}{*}{\multicolumn{2}{|c|}{ Modalité }} & \multicolumn{4}{|c|}{ Activité oxydasique $(\mathrm{u}, \mathrm{O})$} \\
\hline & & $\mathrm{pH}$ & $\begin{array}{l}\text { Valeur } \\
\text { théorique } \\
\text { (VT) }\end{array}$ & $\begin{array}{l}\text { Valeur } \\
\text { mesurée } \\
\text { (VM) }\end{array}$ & $\begin{array}{l}\text { Récupération } \\
(\mathrm{VM} / \mathrm{VT} \times 100)\end{array}$ \\
\hline & Dilution au $1 / 2$ & $\begin{array}{r}8 \\
9 \\
10\end{array}$ & $\begin{array}{l}18,1 \\
16,3 \\
16,1\end{array}$ & $\begin{array}{l}18,0 \\
15,9 \\
14,5\end{array}$ & $\begin{array}{l}99,4 \\
97,6 \\
90,1\end{array}$ \\
\hline & $\begin{array}{l}\text { Dilution au } 1 / 2+\text { ajout } \\
\text { céruloplasmine }(8,7 \text { u. } 0)\end{array}$ & $\begin{array}{r}8 \\
9 \\
10\end{array}$ & $\begin{array}{l}22,4 \\
20,6 \\
20,5\end{array}$ & $\begin{array}{l}22,0 \\
19,5 \\
18,1\end{array}$ & $\begin{array}{l}98,2 \\
94,7 \\
88,3\end{array}$ \\
\hline & $\begin{array}{l}\text { Dilution au } 1 / 2+\text { ajout } \\
\text { céruloplasmine }(17,5 \text { u. } 0)\end{array}$ & $\begin{array}{r}8 \\
9 \\
10\end{array}$ & $\begin{array}{l}16,8 \\
25,0 \\
24,9\end{array}$ & $\begin{array}{l}25,6 \\
23,9 \\
23,5\end{array}$ & $\begin{array}{l}95,5 \\
95,6 \\
94,4\end{array}$ \\
\hline & $\begin{array}{l}\text { Dilution au } 1 / 2+\text { ajout } \\
\text { céruloplasmine }(35 \mathrm{u} .0)\end{array}$ & $\begin{array}{r}8 \\
9 \\
10\end{array}$ & $\begin{array}{l}35,6 \\
33,8 \\
33,6\end{array}$ & $\begin{array}{l}34,5 \\
33,8 \\
33,8\end{array}$ & $\begin{array}{r}96,9 \\
100,0 \\
100,6\end{array}$ \\
\hline & $\begin{array}{l}\text { Dilution au } 1 / 2+\text { ajout } \\
\text { céruloplasmine }(53,8 \text { u.O })\end{array}$ & $\begin{array}{r}8 \\
9 \\
10\end{array}$ & $\begin{array}{l}45,0 \\
43,2 \\
43,0\end{array}$ & $\begin{array}{l}45,6 \\
42,7 \\
42,4\end{array}$ & $\begin{array}{r}101,3 \\
98,8 \\
98,6\end{array}$ \\
\hline & $\begin{array}{l}\text { Dilution au } 1 / 2+\text { ajout } \\
\text { céruloplasmine }(70 \mathrm{u} .0)\end{array}$ & $\begin{array}{r}8 \\
9 \\
10\end{array}$ & $\begin{array}{l}53,1 \\
51,3 \\
51,1\end{array}$ & $\begin{array}{l}55,3 \\
51,6 \\
51,8\end{array}$ & $\begin{array}{l}104,1 \\
100,6 \\
101,4\end{array}$ \\
\hline
\end{tabular}


Dans ces conditions, le meilleur pourcentage moyen de récupération est obtenu à $\mathrm{pH} 8(99,2 \%)$, puis à $\mathrm{pH} 9(97,9 \%)$ et enfin à $\mathrm{pH} 10(95,6 \%)$.

La détermination du $\mathrm{pH}$ réactionnel optimal n'est pas effectuée par une étude classique déterminant les déviations optiqués d'une solution de référence selon divers $\mathrm{pH}$. Nous avons préféré pour notre part retenir le $\mathrm{pH}$ de la solution tamponnée (réactif 1) correspondant aux conditions optimales de linéarité, de répétabilité, de reproductibilité et de récupération, qui sont en fin de compte des paramètres déterminant de la fiabilité d'une méthode.

En conclusion à l'étude concernant le $\mathrm{pH}$ du tampon, on choisit d'effectuer la réaction chez les bovins à $\mathrm{pH} 9$, modalité qui combine au mieux une bonne linéarité, une bonne répétabilité et une bonne récupération. Le même $\mathrm{pH}$ du tampon est choisi pour les ovins, car il entraîne un pH du milieu réactionnel final considéré comme optimal pour cette espèce.

\section{Reproductibilité.}

Pour le pH choisi (9) on étudie le coefficient de variation entre séries (reproductibilité) à partir de 3 plasmas de vache. Pour le plasma « faible " la reproductibilité est de 7,0\% (valeur : 10,0 $\pm 0,7$ u. $0, n=60$ ), pour le plasma " moyen » de $2,6 \%$ (valeur $35,2 \pm 0,9$ u. $0, \mathrm{n}=30$ ) et pour le plasma " fort " de $2,2 \%$ (valeur: $68,1 \pm 1,5$ u. $0, \mathrm{n}=60$ ).

\section{Cinétique de réaction.}

La cinétique de la réaction, mesurée toutes les 10 secondes à $\mathrm{pH} 9$ et pour des activités oxydasiques des plasmas de 0 (blanc de réactif), 14, 46 et $61 \mathrm{u} . \mathrm{O}$ est précisée par la figure 3 . La réaction est linéaire pour le mode opératoire choisi

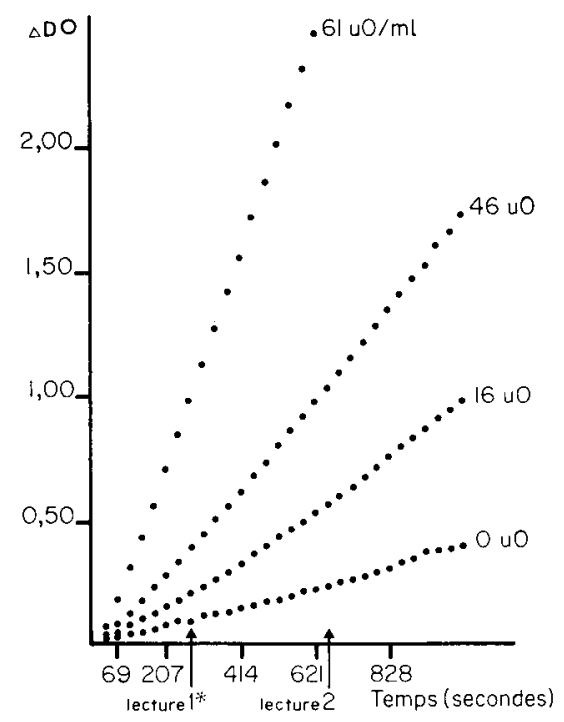

FIG. 3. - Cinétique de développement de la réaction colorée à pH 9 pour des concentrations en céruloplasmine comprises entre 0 et 61 unités Oxydasiques.

* La différence de D.O entre les deux lectures est celle qui permet à I'ISAMAT de calculer les activités oxydasiques. 
(première lecture de D.O à $289 \mathrm{~s}$, deuxième lecture à $634 \mathrm{~s}$ ). Le blanc de réactif montre une dérive également linéaire, ce qui n'est pas gênant dans nos conditions analytiques, puisque la variation de D.O du blanc est déduite automatiquement par l'automate de la variation mesurée pour chaque échantillon.

\section{Modalité de conservation et influence des congélations-décongélations} (tabl. 2).

L'activité oxydasique de la céruloplasmine diminue assez rapidement à température ambiante ( $-12,2 \%$ en $24 \mathrm{~h},-33,3 \%$ en $48 \mathrm{~h})$. Par contre, une conservation à $-20{ }^{\circ} \mathrm{C}$ pendant 1 mois ne diminue l'activité moyenne que de $6,7 \%$. Cette perte d'activité peut être toutefois considérée comme acceptable dans la mesure où la diminution d'activité à $-20^{\circ} \mathrm{C}$ est du même ordre pour les faibles et les fortes valeurs $(-7,3 \%$ pour la valeur minimum, $-6,0 \%$ pour la valeur maximum). Une modalité de conservation courante lors de prélèvements sur le terrain, soit une mise à $4^{\circ} \mathrm{C}$ quelques heures suivie d'une congélation jusqu'à analyse, apparaît donc utilisable pour la détermination de l'activité oxydasique de la céruloplasmine, la conservation pendant $24 \mathrm{~h}$ à $4^{\circ} \mathrm{C} \mathrm{n}^{\prime}$ induisant pas de perte d'activité.

TABLEAU 2

Variations de l'activité oxydasique de la cérulop/asmine plasmatique selon la température et le temps de conservation.

\begin{tabular}{lccr}
\hline & \multicolumn{3}{c}{ Activité oxydasique en u.O (n $=6$ ) } \\
\cline { 2 - 4 } \multicolumn{1}{c}{$\begin{array}{c}\text { Durée et mode de conservation } \\
\text { des échantillons }\end{array}$} & Moyenne & Écart-type & Minimum-maximum \\
\hline Plasma frais & 41,7 & 19,9 & $10,9-67,1$ \\
24 h à température ambiante & 36,6 & 18,7 & $8,0-59,3$ \\
24 h à $4{ }^{\circ} \mathrm{C}$ & 43,2 & 21,8 & $9,5-70,4$ \\
48 h à température ambiante & 27,8 & 15,0 & $5,1-45,7$ \\
48 h à $4{ }^{\circ} \mathrm{C}$ & 39,7 & 21,4 & $6,6-67,1$ \\
$15 \mathrm{j}$ à $-20^{\circ} \mathrm{C}$ & 39,3 & 18,6 & $10,8-63,5$ \\
1 mois à $-20^{\circ} \mathrm{C}$ & 38,9 & 18,4 & $10,1-63,1$ \\
\hline
\end{tabular}

Pour préciser l'influence éventuelle des congélations-décongélations, quatre aliquotes de 6 plasmas ont été congelées à $-20^{\circ} \mathrm{C}$ juste après prélèvement et l'activité oxydasique mesurée après 1 à 4 décongélations étalées sur 4 jours (Les plasmas restaient $1 \mathrm{~h}$ à température ambiante avant recongélation).

L'aliquote subissant une seule congélation-décongélation l'a subie le $4^{e}$ jour, l'aliquote en subissant deux a été décongelée les 1 er et $4^{e}$ jours et ainsi de suite jusqu'à la $4^{\mathrm{e}}$ aliquote, qui a donc été congelée-décongelée chaque jour. Toutes les aliquotes ont été dosées en triple dans trois séries successives immédiatement après la dernière décongélation. Les valeurs obtenues n'étant pas différentes quel que soit le nombre de congélations-décongélations $(39,4 \pm 18,8$ u.0 ; 
$39,7 \pm 18,4$ u. $0 ; 39,7 \pm 19,1$ u. $0 ; 39,7 \pm 18,8$ u. $O$ pour 1 à 4 congélationsdécongélations), on peut conclure à l'absence d'influence de la congélationdécongélation sur l'activité oxydasique de la céruloplasmine.

\section{Comparaison de méthodes.}

Afin d'évaluer la méthode $O X Y$, on a effectué sur plasmas bovin et ovin une comparaison avec les résultats fournis par d'autres méthodes (IDR et CUP). Pour la méthode IDR, les résultats de chaque échantillon ont été calculés par comparaison avec un "pool-témoin ", dont la valeur a été fixée à 200 u.D (unités de Diffusion). Le tableau 3 indique que les valeurs des plasmas utilisés dans les comparaisons ont une plage de variation importante, correspondant pour les valeurs basses à de nets états de carences, et pour les valeurs élevées à des états inflammatoires aigus. Les coefficients de variation apparaissent inférieurs avec la méthode CUP par rapport aux deux autres méthodes. Les coefficients de corrélations calculés entre méthodes (fig. 4 et 5 ) apparaissent de même ordre, quelles que soient les espèces et les méthodes. Ils sont tous élevés (entre 0,944 et 0,968 ) et indiquent une bonne correspondance de la méthode OXY avec les méthodes IDR et CUP.

\section{TABLEAU 3}

Valeurs trouvées selon les méthodes sur les populations de bovins et d'ovins utilisées dans la comparaison entre méthodes.

\begin{tabular}{cccccc}
\hline Méthode & $\begin{array}{c}\text { Nombre } \\
\text { d'échantillons }\end{array}$ & Moyenne & $\begin{array}{c}\text { Coefficient } \\
\text { de variation (\%) }\end{array}$ & Minimum & Maximum \\
\hline $\begin{array}{c}\text { Bovins: } \\
\text { OXY } \\
\text { (u.O) } \\
\text { IDR } \\
\text { (u.D) } \\
\begin{array}{c}\text { CUP } \\
\text { (ug/100 ml) }\end{array}\end{array}$ & 92 & 44,0 & 34,5 & 8,6 & 84,0 \\
\hline $\begin{array}{c}\text { Ovins: } \\
\text { OXY }\end{array}$ & 92 & 169,0 & 35,9 & 44,0 & 365,0 \\
$\begin{array}{c}\text { (u.O) } \\
\text { IDR } \\
\text { (u.D) } \\
\text { CUP }\end{array}$ & 76 & 111,8 & 23,9 & 46,0 & 186,0 \\
$(\mu \mathrm{g} / 100 \mathrm{ml})$ & 76 & 148,8 & 30,7 & 24,0 & 233,0 \\
\hline
\end{tabular}

OXY : céruloplasmine oxydasique; IDR : céruloplasmine par immunodiffusion radiale; CUP : cuprémie par absorption atomique).

\section{Discussion.}

Le $\mathrm{pH}$ du tampon choisi (9) pour les bovins correspond à un $\mathrm{pH}$ réactionnel final de 6,3, qui d'après Calabrese et al. (1983) est le pH réactionnel optimal pour 


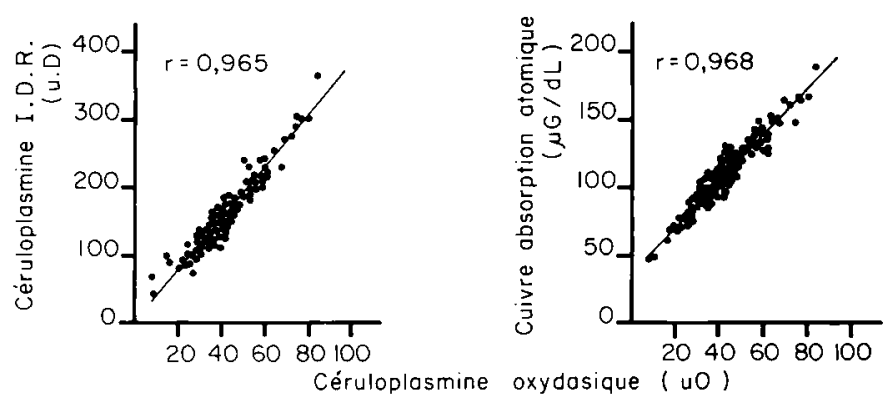

FIG. 4. - Relations - chez les bovins - entre les méthodes de détermination de la céruloplasmine et du cuivre plasmatique ( $\mathrm{n}=92$ ).

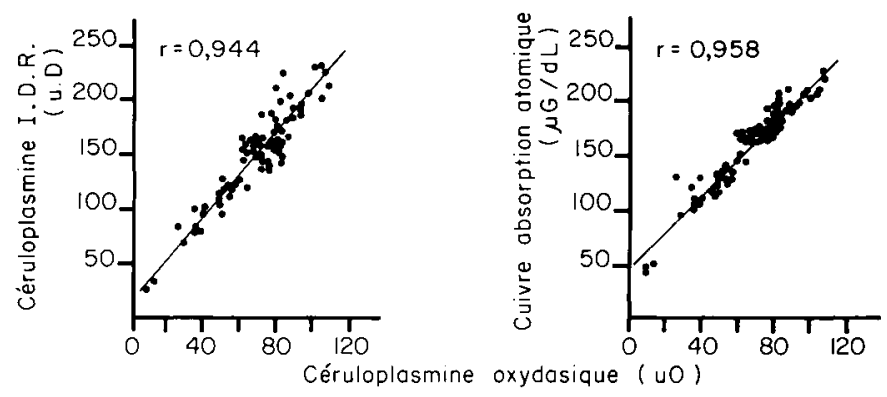

FIG. 5. - Relations - chez les ovins - entre les méthodes de détermination de la céruloplasmine et du cuivre plasmatiques $(n=76)$.

les ovins avec la méthode oxydasique : la méthode que nous avons mise au point peut donc appliquer indifféremment aux bovins et aux ovins. Quant au domaine de linéarité de notre méthode, il apparaît satisfaisant, l'absence de références sur ce point dans la littérature ne nous permettant pas d'effectuer de comparaisons avec d'autres auteurs (la même remarque peut être faite pour les tests de récupération).

Pour ce qui est de la répétabilité de la méthode oxydasique, elle a été testée chez l'homme par Jérôme et Girault (1969), qui ont trouvé 1,4\% et par Sunderman et Nomoto (1970), qui ont trouvé 1,25\%: ces chiffres sont du même ordre que ceux que nous avons mis en évidence $(1,8 \%)$ chez les bovins. La méthode d'immunoprécipitation en milieu liquide donne d'après Buffone et al. (1979) de moins bons résultats, avec une répétabilité de $4,6 \%$ et une reproductibilité de $6,3 \%$. Pour la reproductibilité, elle était de 3,7 \% pour Jérôme et Girault (1969), de $2,8 \%$ pour Sunderman et Nomoto (1970) - chiffres comparables à nos résultats $-(2,6 \%$ de répétabilité pour les valeurs moyennes).

Les coefficients de corrélation ( $r$ ) OXY-CUP sont légèrement supérieurs avec notre méthode $(0,958$ et 0,968$)$ ceux déterminés par Abdel Rahim (1982) sur vaches Zébus ( $r$ moyen de 0,943) et par Osman et al. (1984) sur mouton ( $r$ moyen 
de 0,937$)$. Quant à la corrélation OXY-IDR, elle n'a pas été effectuée dans d'autres travaux; chez l'homme, Ruelland et al. (1982) ont toutefois déterminé des coefficients de corrélation de 0,886 et 0,946 pour des sérums témoins et sérums inflammatoires, entre la méthode oxydasique et une méthode immunologique utilisant l'immunoprécipitation en milieu liquide. En fait, de tels coefficients de corrélation ne peuvent vraisemblablement pas être augmentés de manière significative, car les méthodes comparées sont basées sur des principes réactionnels différents :

- la méthode basée sur I'IDR ou sur une autre technique immunologique évalue la céruloplasmine en tant qu'antigène, mais également une autre protéine, l'apocéruloplasmine, par ailleurs dépourvue d'activité oxydasique (Ruelland et al., 1982) ;

- I'activité oxydasique de la céruloplasmine est dépendante des seuls atomes de cuivre de la protéine existant sous forme oxydée, ainsi que de la richesse du milieu en fer (Foulhoux, 1973) ;

- la méthode CUP dose l'ensemble des atomes de cuivre présents dans le plasma, sous forme liée à la céruloplasmine mais aussi à d'autres protéines et à des acides aminés.

Les modalités de conservation de l'activité oxydasique, sont satisfaisantes vis-à-vis d'une utilisation en routine de la méthode OXY. La miniaturisation de la technique oxydasique permet de travailler avec de faibles consommations de réactifs et de faibles volumes d'échantillon $(12 \mu \mathrm{l})$. L'automatisation réalisée sur l'analyseur Isamat implique en outre une grande vitesse d'analyse des plasmas (environ 500 résultats/jour/opérateur), supérieure à celle des méthodes précédemment publiées (150 déterminations/jour/opérateur en spectrophotométrie d'absorption atomique).

\section{Conclusion.}

Les conditions de dosage que nous avons définies permettent de déterminer l'activité oxydasique de la céruloplasmine plasmatique chez les bovins et les ovins :

- avec une méthode à grand domaine de linéarité et bien reproductible ;

- en utilisant de faibles volumes d'échantillons et de réactifs impliquant un faible coût analytique 11,70 franc/échantillon ; réactifs, consommables et main-d'œuvre compris, année 1985);

- avec une cadence de dosage élevée permettant un faible délai de réponse du laboratoire vis-à-vis d'intervenants de terrain.

Dans ces conditions, un suivi de la nutrition en cuivre peut être réalisé chez les bovins et les ovins à partir de la détermination de l'activité oxydasique de la céruloplasmine. II en est de même pour le dépistage des états inflammatoires existant dans les troupeaux, en associant le dosage de la céruloplasmine à celui d'autres protéines, notamment de l'haptoglobine (Venien, 1982) et à la détermination d'activités enzymatiques plasmatiques traduisant spécifiquement des lésions organiques. 
Remerciements. - Nous remercions D. Levieux (Laboratoire des Maladies nutritionnelles, I.N.R.A., Theix) qui a mis à notre disposition un antisérum spécifique de la céruloplasmine.

\section{Références}

ABDEL RAHIM A. G., 1982. The relationship between the concentrations of serum copper and the activities of the serum enzyme copper oxidase in Sudanese Zebu type dairy cows. Anim. Prod., 18, 53-56.

AUZA N., 1983. Le cuivre chez les ruminants. Une revue. Ann. Rech. vét., 14, 21-37.

BARNOUIN J., QUECHON M., PETIT B., NICOLAS J. A., BROCHART M., 1985. Dominantes pathologiques et gestion sanitaire en élevage ovin de semi-plein-air. Bull. Tech. CRZV Theix - I.N.R.A., 61 (sous presse).

BELLANGER J., LAMAND M., 1975. Méthode de dosage du cuivre et du zinc plasmatiques. Bull. Tech. CRZV TheiX - I.N.R.A., 20, 53-54.

BUFFONE G. J., BRETT E. M., LEWIS S. A., LOSEFSOHN M., HICKS J. M., 1979. Limitations of immunochemical measurement of ceruloplasmin. Clin. Chem., 5, 749-751.

CALABRESE L., CADUOZZO E., GALTIERI A., BELLOCO E., 1983. Sheep ceruloplasmin : isolation and characterisation. Molec. cell. Biochem., 51, 129-132.

CONFORTI A., FRANCO L., MILANINO R., VELO G. P., 1982. Copper and ceruloplasmin (Cp) concentrations during the acute inflammatory process in the rat. Agents and Actions, 12, 303-307.

FOULHOUX P., 1973. La céruloplasmine. Intérêt actuel en biologie et en pathologie. Prod. Prob/. Pharm., 1, 173-190.

GRAFMEYER D., 1978. Etude des performances globales d'après le protocole de la S.F.B.C., 129137. In Mesure des activités enzymatiques en biologie clinique. Ed. S.F.B.C. Lyon.

HOLMBERG C. G., LAURELL C. B., 1951. Investigations in serum copper. III. Ceruloplasmin as an enzyme. Acta. chem. scand., 5, 476.

JÉRÓME H., GIRAULT M., 1969. Dosage de la céruloplasmine. Normalisation des résultats. Ann. Biol. clin., 27, 371-385.

LAMAND M., LEVIEUX D., 1981. Effects of infection on plasma levels of copper and zinc in ewes. Ann. Rech. vét. 12, 133-136.

LAMAND M., PERIGAUD S., BELLANGER J., 1973. Enquête sur la fréquence et la répartition géographique des carences en oligo-éléments en France. Cah. Méd. vét., 42, 155-175.

MANCINI G., CARBONARA A. D., HEREMANS J. F., 1965. Immunochemical quantitation of antigens by single radial immunodiffusion. Immunochemistry, 2, 235-239.

MASON J., LAMAND M., KELLEHER C. A., 1980. The fate of Mo-labelled sodium tétrathiomolybdate after duodenal administration in sheep : the effect on ceruloplasmin diamine oxidase activity and plasma copper. Brit. J. Nutr., 43, 515-523.

OSMAN A. A., ABDEL RAHIM A. G., GAMEEL A. A., BUSHARA H. O., 1984. The relationship between serum copper and zinc concentrations and the activities of the serum enzymes copper oxydase and alkaline phosphatase in sheep infected with Schistosoma bovis and fed on different levels of nutrition. World Rev. anim. Prod., 20, 33-37.

PRESSAC M., VILLEVAL J. L., LEFEBVRE H., AYMARD P., 1983. Evaluation d'un analyseur à transfert ISAMAT. Etude de dosages sériques en cinétique et en point final. Inform. Sci. biol., 9, 21-45.

RABOISSON T., 1985. Contribution à l'étude du diagnostic de l'inflammation chez les ruminants. Th. Méd. vét., Ecole Nat. Vét. Alfort - Fac. Méd. Créteil (sous presse).

RUELLAND A., GOBRON S., CLOAREC L., 1982. Du choix d'une technique de dosage de la céruloplasmine dans le syndrome inflammatoire et au cours du troisième trimestre de la grossesse. Ann. Biol, clin., 40, 11-15.

SUNDERMAN F. W., NOMOTO Jr. S., 1970. Measurement of human serum ceruloplasmin by its p-phenylenediamine oxydase activity. Clin. Chem., 11, 903-910.

VENIEN A., 1982. L'haptoglobine, protéine de l'inflammation. Etude bibliographique et approche expérimentale chez les ruminants. Mém. D.E.A., Univ. Clermont-Ferrand - I.N.R.A., Laboratoire des Maladies Nutritionnelles, $48 \mathrm{pp}$. 